\title{
Étude des caissons romains du lac de Nemi
}

Opération subaquatique 2021

\section{Emmanuel Nantet}

\section{(2) OpenEdition \\ Journals}

Édition électronique

URL : https://journals.openedition.org/baefe/4938

DOI : $10.4000 /$ baefe.4938

ISSN : 2732-687X

Éditeur

ResEFE

\section{Référence électronique}

Emmanuel Nantet, «Étude des caissons romains du lac de Nemi » [notice archéologique], Bulletin archéologique des Écoles françaises à l'étranger [En ligne], Italie, mis en ligne le 24 février 2022, consulté le 26 février 2022. URL : http://journals.openedition.org/baefe/4938 ; DOl : https://doi.org/10.4000/ baefe.4938

Ce document a été généré automatiquement le 26 février 2022.

\section{(c) (i) (9)}

Le Bulletin archéologique des Écoles françaises à l'étranger est mise à disposition selon les termes de la Licence Creative Commons Attribution - Pas d'Utilisation Commerciale - Pas de Modification 4.0 International. 


\section{Étude des caissons romains du lac de Nemi}

Opération subaquatique 2021

\section{Emmanuel Nantet}

\section{NOTE DE L'AUTEUR}

Date précise de l'opération : 7-14 août 2021

Composition de l'équipe de terrain : Emmanuel Nantet, Université de Haïfa ; Rupert

Breitwieser, Université de Salzburg.

Partenariats institutionnels : ASSO, Université de Haïfa, Leon Recanati Institute for Maritime Studies, Université de Salzburg, Université de Vienne, Université Louis-etMaximilien de Munich, CReAAH (UMR 6566).

Établissement éditeur : EFR

Établissements porteurs de l'opération : ASSO, Université de Haïfa

1 Bien que les caissons en bois de Nemi apportent des informations déterminantes pour retracer la mise en valeur du lac à l'époque romaine et pour comprendre les techniques de construction en milieu immergé, ils n'avaient guère attiré l'attention jusqu'à présent. Le lac est surtout renommé pour les deux épaves des bateaux géants bâtis par Caligula. L'assèchement du lac entre 1928 et 1932, sous Mussolini, a permis l'extraction des navires, dans une atmosphère d'exaltation du régime fasciste, puis leur exposition dans un musée, le Museo Nazionale delle Navi Romane, spécifiquement bâti pour eux et inauguré en 1936. C'est à cette occasion que les structures en bois autour du lac ont été mises au jour. En dépit du faible intérêt suscité par leur découverte, la publication des navires, assurée par Guido Ucelli avec une grande rigueur ${ }^{1}$, comporte des dessins précis sur leur localisation et l'assemblage des sablières qui composent les "palissades » observées sur certaines rives du lac, notamment dans sa partie méridionale.

2 La perte des épaves en 1944, à la fin de la Seconde Guerre Mondiale, a marqué un tournant dans les études sur Nemi. Malgré leur destruction, elles continuent de susciter 
une ferveur populaire, parfois complètement déconnectée de la réalité archéologique. La récente prospection instrumentée, bien qu'elle n'ait pas permis l'identification d'une «troisième épave », n'a pas mis fin aux spéculations. Quoi qu'il en soit, les études archéologiques menées sur la commune portent désormais sur divers sites autour du lac, qu'il s'agisse de la villa au sud-ouest ${ }^{2}$ ou du sanctuaire de Diane au nord-est. Les fouilles menées sur ce dernier de 1989 à 2009, d'abord sous la direction de Giuseppina Ghini, rejoints par Paolo Braconi, Filippo Coarelli et Francesca Diosono, se sont ainsi concentrées sur la terrasse et le nymphée ${ }^{3}$. L'étude des lampes retrouvées dans les eaux $\mathrm{du}$ lac lors de son assèchement montre que des rituels s'y déroulaient à l'époque impériale ${ }^{4}$.

3 La prospérité du lieu, apprécié des élites romaines, reposait notamment sur la mise en valeur du lac, formé dans le cratère d'un volcan, dont l'eau, qui irriguait les environs, était contrôlé grâce un émissaire long de $1653 \mathrm{~m}^{5}$. La construction des "palissades " pourrait donc avoir eu pour fonction de renforcer les berges du lac. La datation au radiocarbone d'un échantillon prélevé sur un poteau, apparu lors d'une période de sécheresse qui a provoqué l'abaissement du niveau du lac, a indiqué une date entre 170 et 45 avant n. è. ${ }^{6}$ Plus récemment, les travaux menés par le groupe ROMACONS (Roman Maritime Concrete Study) ont proposé d'identifier cette structure comme un caisson ${ }^{7}$. La construction en caisson relève d'une technique qui permet de bâtir des structures en milieu immergé, à laquelle il faut attribuer l'aménagement des vastes ports de l'époque impériale. La complexité de la technique est apparue à l'issue des fouilles menées dans le port de Césarée, en Israël, dans les années 1980. En effet, la découverte d'un bloc de ciment coulé dans un caisson, gisant par plusieurs mètres de fond, montrait que celui-ci n'avait pas pu être mis en œuvre selon les techniques décrites par Vitruve ${ }^{8}$. La comparaison avec de nombreux autres sites portuaires révélant l'usage de la même technique de construction a montré que la question devait être étendue à l'ensemble des ports de la Méditerranée romaine, ce qui a permis d'établir une typologie de caissons ${ }^{9}$. Toutefois, si l'ouvrage détaille les nombreuses structures en ciment bâties dans les ports de la Méditerranée romaine, il se focalise sur les ports maritimes du Haut-Empire. En d'autres termes, il exclut les attestations éventuellement plus précoces ou plus tardives de cette technique. Il ne porte pas non plus sur les structures lacustres, qui répondent à des contraintes différentes et requièrent par conséquent des techniques de construction adaptées à leur environnement spécifique. Ainsi, les structures portuaires du lac Nemi ne sont guère abordées dans cet ouvrage. C'est en fait à l'occasion des fouilles franco-israéliennes menées sur les rives du lac de Tibériade, en Israël, que le parallèle a été établi, puisque ce dernier site comporte des structures en ciment, peut-être d'époque byzantine, également bâties dans un caisson de bois (ISF Grant No. 512/19). Dans un lac, l'absence de vagues et de courants constituent en effet des conditions différentes des milieux maritimes ou fluviaux lors de la construction d'un port.

4 En d'autres termes, les structures ligneuses observées à Nemi constitueraient une attestation particulièrement ancienne de la construction en caisson. Leur étude permettrait ainsi de mieux saisir l'évolution de cette technique de construction et notamment de déterminer son origine. Les caissons servaient avant tout à renforcer les rives du lac, mais le recours à cette technique interroge. La présence de structures portuaires aussi imposantes, que l'on trouve habituellement dans les grands ports $\mathrm{du}$ monde romain et qui s'étendraient sur une large partie des rives du lac, est remarquable pour un plan d'eau de dimensions relativement modestes. Elle s'explique 
naturellement par l'implication des élites romaines et du pouvoir impérial. Ainsi, une datation plus assurée de ces caissons permettrait de comprendre comment ces structures portuaires s'articulent avec le reste des vestiges mis au jour dans le lac ou sur son pourtour, à commencer par les navires géants, la villa romaine et le sanctuaire de Diane.

5 Une première plongée a donc été effectuée en juin 2019 afin d'observer quelques poteaux qui dépassaient des fonds du lac le long de sa rive méridionale, en contrebas du cimetière de Genzano. Elle a été suivie d'une opération subaquatique, menée du 7 au 14 août 2021, sous la direction d'Emmanuel Nantet, avec le concours des universités de Haïfa, Munich et Vienne, ainsi que du Leon Recanati Institute for Maritime Studies, de l'ASSO, du CReAAH, de l'ARESMAR et de l'École française de Rome.

Nemi : Poteau 34 en cours de fouille.

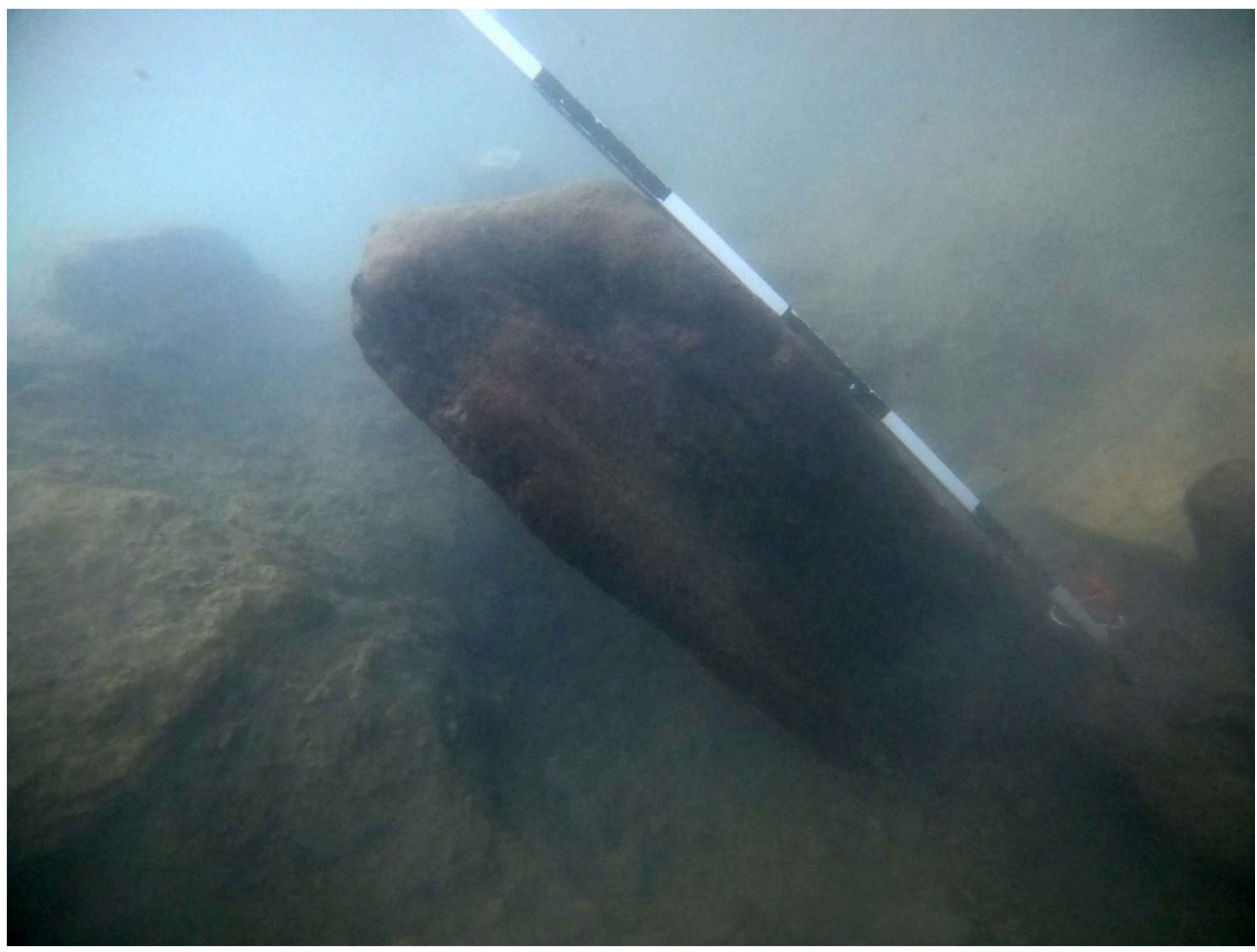

Jehan Marie.

La prospection a ainsi permis d'identifier des dizaines de poteaux de bois, reposant par une trentaine de centimètres de profondeur et dont le sommet dépassait parfois de la surface de l'eau. Les poteaux étaient disposés sur deux lignes parallèles au bord du lac. La surface des bois était détériorée, ce qui a empêché toute étude tracéologique et rendu difficile la compréhension de leur assemblage. L'opération a donné lieu à un échantillonnage systématique de tous les bois mis au jour afin de déterminer les essences dans lesquelles ils ont été débités. Par ailleurs, quelques bois ont été sectionnés afin de mener une analyse dendrochronologique.

7 La prospection n'a pas permis de déterminer avec certitude si la structure étudiée durant le mois d'août 2021 correspond à l'une des «palissades » décrite par Guido Ucelli ${ }^{10}$. Les rives du lac ont considérablement changé au cours depuis les années 1930 et il est parfois difficile de suivre le tracé des structures indiqué sur le plan livré par 
l'archéologue italien. Quoi qu'il en soit, l'extension de la prospection subaquatique à l'ensemble de la rive méridionale du lac a permis d'identifier quelques autres poteaux, mais ceux-ci présentent des caractéristiques différentes qui laissent envisager des datations plus récentes.

\section{BIBLIOGRAPHIE}

BILDE 2005

Pia Guldager Bilde, « The Roman villa by Lake Nemi: from nature to culture - between private and public », in Barbro Santillo Frizell et Allan Klynne (éd.), Roman villas around the urbs. Interaction with landscape and environment, Rome, Swedish Institute, 2005, p. 211-219.

BRACONI et al. 2014

Paolo Braconi et al. (éd.), Il santuario di Diana a Nemi. Le terrazze e il ninfeo. Scavi 1989-2009, Rome, 2014.

BRACONI, DIOSONO 2012

Paolo Braconi, Francesca Diosono, « Il ninfeo di Caligola nel santuario di Nemi ed il controllo delle acque del lago », Forma Urbis XVII, 12, 2012, p. 40-43.

BRANDON 2014a

Christopher Brandon, "Maritime Concrete in the Mediterranean World », in

Christopher Brandon, Robert L. Hohlfelder, Marie D. Jackson et John P. Oleson (éd.), Building for Eternity. The History and Technology of Roman Concrete Engineering in the Sea, Oxford, Oxbow Books, 2014, p. 121-140.

BRANDON 2014b

Chirstopher Brandon, « Roman Formwork Used for Underwater Concrete Construction », in Christopher Brandon, Robert L. Hohlfelder, Marie D. Jackson et John P. Oleson (éd.), Building for Eternity. The History and Technology of Roman Concrete Engineering in the Sea, Oxford, Oxbow Books, 2014, p. 189-222.

BRANDON et al. 2014

Christopher Brandon et al., Building for Eternity. The History and Technology of Roman Concrete Engineering in the Sea, Oxford, Oxbow Books, 2014.

CASTELLANI, ALOI 1991

Vittorio Castellani, Vittoria Aloi, « Notes on the ancient emissary of Lake Nemi », Atti 3rd International Symposium on Underground Quarries, 10-14 luglio 1991, Naples, Club Alpino Italiano, 1991, p. 206-220.

CASTELLANI et al. 2002

Vittorio Castellani et al., « Nemi. Nuove evidenze per l'antica storia dell'emissario nemorense (Roma) », Opera Ipogea 2, 2002, p. 51-58.

CASTELLANI et al. 2003

Castellani V. et al., « L'emissario del Lago di Nemi. Indagine topografico-strutturale », Opera Ipogea 2/3, 2003, p. 2-76. 
DIOSONO, CINAGLIA 2016

Francesca Diosono, Tiziano Cinaglia, « Light on the water: Ritual deposit of lamps in Lake Nemi », Journal of Roman Archaeology 29, 2016, p. 451-468.

GHINI 1996

Giuseppina Ghini, « Prospezioni Subacquee nei Laghi Albano e Nemorense », Bollettino di

Archeologia Subacquea 1-2, 1996, p. 184-196.

MOLTESEN, POULSEN 2010

Mette Moltesen, Birte Poulsen (éd.), A Roman villa by Lake Nemi. The finds. The Nordic excavations by Lake Nemi, loc. S. Maria (1998-2002), Rome, Edizioni Quasar, 2010.

UCELLI 1952

Guido Ucelli, Le Navi di Nemi, Rome: Libreria dello Stato, 1952 (2e éd., réimpr. 1983).

\section{NOTES}

1. UCELLI 1952.

2. BILDE 2005 ; MOLTESEN, POULSEN 2010.

3. BRACONI et al. 2014.

4. DIOSONO, CINAGLIA 2016.

5. CASTELLANI, ALOI 1991 ; CASTELLANI et al. 2002 ; CASTELLANI et al. 2003.

6. GHINI 1996, p. 192-93.

7. BRANDON 2014a, p. 127.

8. Vitruve V, 12, 2-6.

9. BRANDON 2014 b.

10. UCELLI 1950, p. 121, fig. 125.

\section{INDEX}

Thèmes : EFA

chronologie https://ark.frantiq.fr/ark:/26678/pcrtQRUOmOfGGo, https://ark.frantiq.fr/ark:/ 26678/pcrt0LUUg09UTA

lieux https://ark.frantiq.fr/ark:/26678/pcrtVwItl7UVbh sujets https://ark.frantiq.fr/ark:/26678/pcrtFnKonRZjWY, https://ark.frantiq.fr/ark:/26678/ pcrtQNxYz6P6Mh, https://ark.frantiq.fr/ark:/26678/pcrtolEegLdQOD, https://ark.frantiq.fr/ ark:/26678/pcrtMr3tb79bV8, https://ark.frantiq.fr/ark:/26678/crtzz3k12Au4F, https:// ark.frantiq.fr/ark:/26678/pcrtPZwqrmWIr4

Année de l'opération : 2021 
AUTEURS

EMMANUEL NANTET

Université de Haïfa

DIRECTEURFOUILLES_DESCRIPTION

EMMANUEL NANTET

Université de Haïfa 